\title{
Universal Parametric Correlations of Eigenvalues of Random Matrix Ensembles
}

\author{
Kasper Juel Eriksen ${ }^{\dagger}$ and Yang Chen \\ Department of Mathematics, Imperial College \\ 180 Queen's Gate, London SW7 2BZ, U K \\ $\dagger$ Ørsted Laboratory, Niels Bohr Institute \\ H.C.Ø. Universitetsparken 5, 2100 Kbh. Ø, Denmark
}

March 22, 2017

\begin{abstract}
Eigenvalue correlations of random matrix ensembles as a function of an external (parametric) perturbation are investigated via the Dyson Brownian Motion model in the situation where the level density has a hard edge singularity. By solving a linearized hydrodynamical equation, a universal dependence of the density-density correlator on the external field is found. As an application we obtain a formula for the variance of linear statistics with the parametric dependence exhibited as a Laplace transform.
\end{abstract}




\section{Introduction.}

Eigenvalues of $N \times N$ matrices can be viewed as energy levels $\left(x_{a}, a=1, \cdots, N\right)$ of an effective Hamiltonian

$$
H_{0}=\sum_{a} x_{a} n_{a}
$$

where $n_{a}$ are the occupation at level $a$. A proposal by Wigner 11 is that the levels are drawn from an ensemble of matrices, and when restricted to the eigenvalues has the joint probability distribution,

$$
P\left(x_{1}, \cdots, x_{N}\right) \prod_{a=1}^{N} d x_{a}=C_{N} \mathrm{e}^{-\beta W\left(x_{1}, \cdots, x_{N} ; u\right)} \prod_{a=1}^{N} d x_{a}
$$

where

$$
W\left(x_{1}, \ldots, x_{N} ; u\right)=-\sum_{a<b} \ln \left|x_{a}-x_{b}\right|+\sum_{a} u\left(x_{a}\right)
$$

$C_{N}$ is a constant and $\beta=2,1,4$ describes ensembles with unitary, orthogonal and symplectic symmetries. The density of levels is defined by

$$
\sigma(x):=\left\langle\sum_{a} \delta\left(x-x_{a}\right)\right\rangle_{\mathrm{eq}}
$$

where $\langle\cdots\rangle_{\text {eq }}$ is an average with weight $P$ of Eq. (2). For $u(x)=x^{2}$ and $x_{a}$ supported on the real line, $\sigma$ is given by the Wigner semi-circular law[1] in the limit of large $N$,

$$
\sigma_{\mathrm{W}}(x) \sim \sqrt{N-x^{2}}, \quad x \in(-\sqrt{N}, \sqrt{N}) .
$$

Other ensembles arising from transport in disordered electronic systems [2], have $x_{a}$ supported on the right half line with different confining potentials, $u(x)$. For $u(x)=x^{\alpha}$ and $\alpha>1 / 2$ one finds the eigenvalue density[3], 4];

$$
\sigma(x) \sim \sqrt{\frac{N}{x}}, \quad x \ll N
$$


is universal near the "hard edge" [5], in the sense that it is independent of $\alpha$. It can be shown that for $0<\alpha<1 / 2, \sigma(x) \sim 1 / x^{1-\alpha} \llbracket$. In this paper we shall be interested in the response of the levels when $H_{0}$ is perturbed by an external potential. Of particular interest is the eigenvalue correlator as a function of the external potential. This problem was first studied in the context of the energy eigenvalues distribution of a disordered metallic ring subjected to an external magnetic field using diagrammatic techniques [6], Jand it was found that the eigenvalue correlations are universal after an appropriate rescaling. These results were later reproduced in [7] using the Brownian motion model of Dyson[ [8] in the hydrodynamical approximation. Exact correlations for all strengths of the perturbation were obtained in [9] using the method of supersymmetry pioneered by Efetov[10]. All of the above results are valid in the bulk of the spectrum where the density is uniform; $\sigma_{\text {bulk }}=\sigma_{\mathrm{W}}(0) \sim$ constant.

The phenomenological theory proposed by Dyson[8] interprets the eigenvalues $x_{a}$ as positions of classical particles which are governed by an over-damped Langevin equation subjected to Gaussian random force $f_{a}(\tau)$,

$$
\gamma \frac{d x_{a}}{d \tau}=-\frac{\partial W}{\partial x_{a}}+f_{a}(\tau)
$$

where $\gamma$ is the friction coefficient,

$$
\overline{f_{a}(\tau)}=0, \quad \overline{f_{a}(\tau) f_{b}\left(\tau^{\prime}\right)}=\frac{2 \gamma}{\beta} \delta_{a b} \delta\left(\tau-\tau^{\prime}\right),
$$

and $\tau$ is related to the strength of the perturbation, $X$. Since the $x_{a}$ 's undergo a Brownian motion it is to be expected that $X^{2} \propto \tau$ [8, [1]. A Fokker-Planck equation that describes

\footnotetext{
${ }^{1}$ More precisely, the density of state-density of state correlation function at different fluxes.
} 
the "time" dependent joint probability distribution can be derived, and reads

$$
\gamma \frac{\partial}{\partial \tau} P\left(x_{1}, \cdots, x_{N}, \tau\right)=\sum_{a} \frac{\partial}{\partial x_{a}}\left[\frac{\partial W}{\partial x_{a}}+\beta^{-1} \frac{\partial}{\partial x_{a}}\right] P\left(x_{1}, \cdots, x_{N}, \tau\right), \quad \tau>0
$$

subjected to the initial condition;

$$
P\left(x_{1}, \cdots, x_{N}, 0\right)=\prod_{a=1}^{N} \delta\left(x_{a}-x_{a}^{0}\right)
$$

where $x_{a}^{0}$ is the initial position of particle $a$. The stationary solution of the Fokker-Planck equation is

$$
P\left(x_{1}, \cdots, x_{N}, \infty\right)=C_{N} \mathrm{e}^{-\beta W}
$$

It was shown by Dyson 8 that the time dependent density,

$$
\sigma(x, \tau)=\left\langle\sum_{a} \delta\left(x-x_{a}(\tau)\right)\right\rangle_{\tau}
$$

$\left[\right.$ here $\langle\cdots\rangle_{\tau}$ denotes average with "time-dependent" weight given in Eq.(9)] satisfies a non-linear conservation law in the "hydrodynamical" approximation. The non-equilibrium density $\sigma(x, \tau)$ evolves in $\tau$ according to

$$
\frac{\partial}{\partial \tau} \sigma(x, \tau)=\frac{\partial}{\partial x}\left(\sigma(x, \tau) \frac{\partial}{\partial x} \Psi\right)
$$

where

$$
\Psi(x, \tau)=u(x)-\int_{K} d y \sigma(y, \tau) \ln |x-y|+\left(\frac{1}{\beta}-\frac{1}{2}\right) \ln [\sigma(x, \tau)] .
$$

Note that the "time" dependent density is normed to $N ; \int_{K} d x \sigma(x, \tau)=N$, where $K$ is the interval on which the levels are supported.

The solution to Eq.(12) with Eq.(13), will enable us to determine the parametric $(\tau)$ dependence of quantities related to the eigenvalues $x_{a}$. 


\section{Linearization.}

The stationary density, $\sigma(x):=\sigma(x, \infty)$, of the non-linear diffusion equation satisfies a self-consistent Hückel type equation,

$$
u(x)-\int_{K} d y \sigma(y) \ln |x-y|+\left(\frac{1}{\beta}-\frac{1}{2}\right) \ln [\sigma(x)]=A=\text { constant }
$$

This suggests that for sufficiently long time円, we split the non-equilibrium density into an equilibrium part plus a small perturbation, $\rho(x, \tau)$;

$$
\sigma(x, \tau)=\sigma(x)+\rho(x, \tau)
$$

where $\sigma(x)$ the equilibrium density. Substituting Eq.(15) into Eq.(12) and discarding all terms of $O\left(\rho^{2}\right)$, gives

$$
\frac{\partial}{\partial \tau} \rho(x, \tau)=-\frac{\partial}{\partial x} J(x, \tau)
$$

where the "particle" flux is

$$
J(x, \tau):=\frac{\sigma(x)}{\gamma} \frac{\partial}{\partial x} \int_{K} \rho(y, \tau) \ln |x-y| d y
$$

This is unlike the ordinary diffusion equation in that the particle flux requires the entire distribution, $\rho$, to specify its value at one point. As an example, we consider the Gaussian ensembles with $u(x)=x^{2}$, and $K=(-\sqrt{N}, \sqrt{N})$. In the $N \rightarrow \infty$ limit, and scaling into the bulk $x \ll N$, where the density is uniform, $\sigma(x)=D=$ constant, the diffusion equation becomes,

$$
\frac{\partial}{\partial \tau} \rho(x, \tau)=-\frac{D}{\gamma} \frac{\partial^{2}}{\partial x^{2}} \int_{-\infty}^{+\infty} d y \rho(y, \tau) \ln |x-y| .
$$

\footnotetext{
${ }^{2}$ More precisely $D \tau / \gamma L \gg 1$, where $L$ is the interval over which the density extends[8].
} 
This is converted into

$$
\frac{\partial}{\partial \tau} \bar{\rho}_{k}(\tau)=-\frac{\pi D}{\gamma}|k| \bar{\rho}_{k}(\tau)
$$

via a Fourier transform [8, [7], $\bar{\rho}_{k}(\tau)=\int_{-\infty}^{+\infty} d x \mathrm{e}^{i k x} \rho(x, \tau)$, with the solution;

$$
\bar{\rho}_{k}(\tau)=\bar{\rho}_{k}\left(\tau^{\prime}\right) \mathrm{e}^{-\frac{\pi D}{\gamma}|k|\left(\tau-\tau^{\prime}\right)}, \quad \tau \geq \tau^{\prime}
$$

From the $\tau$ dependence of $\bar{\rho}_{k}$, we can infer the $\tau$ dependence of

$$
\begin{aligned}
\operatorname{Corr}(x, y, \tau) & :=\langle\sigma(x, \tau) \sigma(y, 0)\rangle_{\mathrm{eq}}-\langle\sigma(x, \tau)\rangle_{\mathrm{eq}}\langle\sigma(y, 0)\rangle_{\mathrm{eq}} \\
& =\int_{-\infty}^{+\infty} \frac{d k}{2 \pi} \int_{-\infty}^{+\infty} \frac{d p}{2 \pi} \mathrm{e}^{-i k x-i p y-\frac{\pi D}{\gamma}|k| \tau} \operatorname{Corr}(p, k),
\end{aligned}
$$

where

$$
<\phi>\mathrm{eq}:=\left(\prod_{a} \int_{-\infty}^{+\infty} d x_{a}^{0}\right) P\left(x_{1}^{0}, \cdots, x_{N}^{0}\right) \phi\left(x_{1}^{0}, \cdots, x_{N}^{0}\right)
$$

denotes an average over the initial condition. Here $\operatorname{Corr}(p, k)$ is the Fourier transform of the equilibrium density-density correlation function [1]; $\operatorname{Corr}(x-y)=-\frac{1}{\pi^{2} \beta} \frac{1}{(x-y)^{2}}, x \neq y$. 田

$$
\operatorname{Corr}(p, k)=\int_{-\infty}^{+\infty} d x \int_{-\infty}^{+\infty} d y \mathrm{e}^{i k x+i p y} \operatorname{Corr}(x-y)=\frac{2}{\pi \beta} \delta(k+p)|k|
$$

Therefore,

$$
\operatorname{Corr}(x, y, \tau)=\frac{1}{\beta \pi^{2}} \Re \frac{1}{\left[\frac{D \pi \tau}{\gamma}+i(x-y)\right]^{2}}
$$

is the universal "time" dependent density-density correlation function when $\tau$ is measured in units of $\frac{\gamma}{\pi D}$ 6, 7, 9]

\footnotetext{
${ }^{3}$ The exact density-density correlation function for the Gaussian ensemble $(\beta=2$, and $x \neq y)$ is $-\left[\frac{\sin \pi(x-y)}{\pi(x-y)}\right]^{2}$ [1 . The hydrodynamical approximation essentially replaces $[\sin \pi(x-y)]^{2}$ by $1 / 2$.

$4[9]$ gives the exact result for all $\tau \geq 0$ and $\beta=2,1,4$.
} 


\section{Hard edge correlations.}

We shall now focus our attention on ensembles where the eigenvalues are supported on the right half-line and $\sigma(x)$ has the universal square root singularity at the origin.

$\operatorname{Corr}(x, y, \tau)$ and $\operatorname{Int}(x, y, \tau)--$ - the twice integrated version of $\operatorname{Corr}(x, y, \tau)$ are derived in Section 3. These are applied to determine the parametric dependence of the variance of arbitrary linear statistics, given by Eqs. (51) and (54). As an application, we compute in Section 4 the variance of $\sum_{a}\left[1+x_{a}(\tau)\right]^{-1}$, which gives the conductance fluctuation of a quasi-one dimensional disordered system as a function of the external perturbation. According to the Landauer formula, the conductance, $g$, is $\sum_{a}\left[1+x_{a}\right]^{-1}$. [2]

We have therefore solved in the hydrodynamical approximation the problem posed in[7] for the case where the equilibrium eigenvalue density displays a hard edge singularity; $\sigma(x) \sim$ $D / \sqrt{x}$. Since translational invariance is no longer valid, results obtained in the bulk scaling limit of the Gaussian ensembles (with $u(x)=x^{2}$ ) are no longer applicable here[12]. For example, the gap formation probability, $E_{\beta}(0, s)$, of the Laguerre ensembles (with $u(x)=$ $x-\alpha \ln x, x>0, \alpha>-1$.) is distinct from that of the Gaussian ensembles[5, 13, 14]. We expect $\operatorname{Corr}(x, y, \tau)$ to have distinct "time" decay modes from that found in the bulk scaling case Đ.

\footnotetext{
${ }^{5}$ An application of a dimensional argument on Eq. (18), obtained by scaling into the bulk of the spectrum, shows that the typical distance covered by a diffusing particle over time $t$ is $|x| \sim t$, which is "faster" then Einstein diffusion; $|x| \sim \sqrt{t}$. Looking ahead to Eq. (24) the same analysis shows that $|x| \sim t^{2 / 3}$. This suggests that particle transport near the hard edge is intermediate between ballistic motion and classical diffusion.
} 
The diffusion equation now reads

$$
\frac{\partial \rho(x, t)}{\partial t}=-\frac{\partial}{\partial x}\left(\frac{1}{\sqrt{x}} \mathrm{P} \int_{0}^{\infty} d y \frac{\rho(y, t)}{x-y}\right),
$$

where $D \tau / \gamma=t$. [We have written $\rho(x, \tau)=\rho(x, t)$.] With the ansatz $\rho(x, t) \propto \rho(x, \lambda) \mathrm{e}^{-\lambda t}$, Eq.(24) becomes,

$$
\lambda \rho(x, \lambda)=\frac{\partial}{\partial x}\left(\frac{1}{\sqrt{x}} \int_{0}^{\infty} d y \frac{\rho(y, \lambda)}{x-y}\right), \quad \lambda>0 .
$$

The boundary condition on $\rho(x, \lambda)$ is such that the particle flux vanishes at the boundaries; $\lim _{x \rightarrow 0} J(x, \lambda)=0$, and $\lim _{x \rightarrow \infty} J(x, \lambda)=0$. The boundary condition at $x=0$ reads,

$$
\lim _{x \rightarrow 0} \frac{1}{\sqrt{x}} \mathrm{P} \int_{0}^{\infty} \frac{\rho(y, \lambda)}{x-y} d y=0
$$

Since $\mathrm{P} \int_{0}^{\infty} \frac{1}{\sqrt{y}(x-y)} d y=0, \quad x>0$, we find it convenient to write $\rho(x, \lambda)$ as

$$
\rho(x, \lambda)=\tilde{\rho}(x, \lambda)+\frac{\operatorname{cst}(\lambda)}{\sqrt{x}},
$$

where $\tilde{\rho}(x, \lambda)$ fulfills a stronger condition,

$$
\int_{0}^{\infty} d y \frac{\tilde{\rho}(y, \lambda)}{y}=0
$$

and $\operatorname{cst}(\lambda)$ to be determined later is a function of $\lambda$ only. In appendix $\mathrm{A}$ it is shown that the final solution (Eq. (42)) fulfills the boundary condition (26). The term $\frac{\operatorname{cst}(\lambda)}{\sqrt{x}}$ is similar to the equilibrium solution and we conjecture that the solution for a general hard edge density always has this structure. We now go on to solve Eq. (25). This can be accomplished by "un-folding" the half-line into the real-line 15. With the change of variables, $v=\sqrt{y}, u=\sqrt{x}$, we have

$$
\begin{aligned}
\int_{0}^{\infty} d y \frac{\rho(y, \lambda)}{x-y} & =\int_{0}^{\infty} d y \frac{\tilde{\rho}(y, \lambda)}{x-y}=\int_{0}^{\infty} d v \frac{2 v \tilde{\rho}\left(v^{2}, \lambda\right)}{u^{2}-v^{2}} \\
& =\int_{0}^{\infty} d v \frac{\tilde{\rho}\left(v^{2}, \lambda\right)}{u-v}+\int_{0}^{\infty} d v \frac{-\tilde{\rho}\left(v^{2}, \lambda\right)}{u+v}
\end{aligned}
$$


Now introduce an odd function of $v$,

$$
\begin{aligned}
& \tilde{\rho}_{1}(v, \lambda) \equiv \tilde{\rho}\left(v^{2}, \lambda\right), \quad v>0 \\
& \tilde{\rho}_{1}(v, \lambda) \equiv-\tilde{\rho}\left(v^{2}, \lambda\right), \quad v<0
\end{aligned}
$$

and find

$$
\begin{aligned}
& \int_{0}^{\infty} d y \frac{\rho(y, \lambda)}{x-y}=\int_{0}^{\infty} d v \frac{\tilde{\rho}_{1}(v, \lambda)}{u-v}+\int_{0}^{\infty} d v \frac{\tilde{\rho}_{1}(-v, \lambda)}{u+v} \\
= & \int_{0}^{\infty} d v \frac{\tilde{\rho}_{1}(v, \lambda)}{u-v}+\int_{-\infty}^{0} d v \frac{\tilde{\rho}_{1}(v, t)}{u-v}=\int_{-\infty}^{+\infty} d v \frac{\tilde{\rho}_{1}(v, \lambda)}{u-v} .
\end{aligned}
$$

With Eq. (33) and Eq. (27) the diffusion equation, Eq. (25), becomes,

$$
\frac{\operatorname{cst}(\lambda)}{u}+\lambda \tilde{\rho}_{1}(u, \lambda)=\frac{1}{2 u} \frac{\partial}{\partial u}\left(\frac{1}{u} \int_{-\infty}^{+\infty} d v \frac{\tilde{\rho}_{1}(v, \lambda)}{u-v}\right) .
$$

Although Eq. (34) is derived for $u>0$ it is also valid for $u<0$. This can be seen from the fact that $\tilde{\rho}_{1}$ is an odd function of $u$. To proceed further, we introduce the even function,

$$
\tilde{\rho}_{2}(u, \lambda):=\frac{\tilde{\rho}_{1}(u, \lambda)}{u}
$$

and with its aid we find $\tilde{\rho}_{2}(u, \lambda)$ satisfies,

$$
2 \lambda \operatorname{cst}(\lambda)+2 \lambda u^{2} \tilde{\rho}_{2}(u, \lambda)=\frac{\partial}{\partial u} \int_{-\infty}^{+\infty} d v \frac{\tilde{\rho}_{2}(v, \lambda)}{u-v}
$$

We have made use of the flux condition at origin, Eq. (28),

$$
\int_{-\infty}^{+\infty} d u \tilde{\rho}_{2}(u, \lambda)=2 \int_{0}^{\infty} d u \tilde{\rho}_{2}(u, \lambda)=0
$$

to arrive at Eq. (36). This accomplishes the un-folding of half-line diffusion equation onto the real line. Clearly, Eq. (36) can be be solved by a Fourier transformation.

Eq. (37) implies that $\tilde{\rho}_{2}(u, \lambda)$ is an oscillatory function of $u$ and its Fourier transform,

$$
\rho_{2}(k, \lambda):=\int_{-\infty}^{+\infty} d u \mathrm{e}^{i k u} \tilde{\rho}_{2}(u, \lambda)
$$


vanishes at $k=0$. A further condition is that $\rho_{2}$ is even in $k$.

Once $\rho_{2}(k, \lambda)$ is found, the original density can be recovered by standard inversion formulas;

$$
\frac{\tilde{\rho}(x, \lambda)}{\sqrt{x}}=\frac{1}{\pi} \int_{0}^{\infty} d k \cos (k \sqrt{x}) \rho_{2}(k, \lambda) .
$$

A simple calculation shows that the transformed density, $\rho_{2}(k, \lambda)$, satisfies an Airy equation with a point source,

$$
\frac{d^{2}}{d k^{2}} \rho_{2}(k, \lambda)+\frac{\pi|k|}{2 \lambda} \rho_{2}(k, \lambda)=2 \pi \operatorname{cst}(\lambda) \delta(k)
$$

The solution of this is a linear combination of $\mathrm{Ai}\left(-(\pi / 2 \lambda)^{1 / 3}|k|\right)$ and $\mathrm{Bi}\left(-(\pi / 2 \lambda)^{1 / 3}|k|\right)$, and reads up to a constant,

$$
\begin{aligned}
\rho_{2}(k, \lambda) & =3^{7 / 6} \Gamma(2 / 3)\left(2 \lambda \pi^{5}\right)^{1 / 6}\left[\operatorname{Bi}(0) \operatorname{Ai}\left(-\left(\frac{\pi}{2 \lambda}\right)^{1 / 3}|k|\right)-\operatorname{Ai}(0) \operatorname{Bi}\left(-\left(\frac{\pi}{2 \lambda}\right)^{1 / 3}|k|\right)\right] \\
& =\pi \sqrt{|k|} J_{1 / 3}\left(\frac{2}{3}\left(\frac{\pi}{2 \lambda}\right)^{1 / 2}|k|^{3 / 2}\right)
\end{aligned}
$$

where we have made use of $\operatorname{Ai}(0)=\frac{3^{-2 / 3}}{\Gamma(2 / 3)}, \operatorname{Bi}(0)=\frac{3^{-1 / 6}}{\Gamma(2 / 3)}$ and the relations between $\operatorname{Ai}(-x), \operatorname{Bi}(-x)$ and $J_{ \pm 1 / 3}\left(\frac{2}{3} x^{3 / 2}\right)$ to arrive at Eq. (41). Note that $\rho_{2}(k, \lambda)$ vanishes at $k=0$ and is even in $k$. We now use the jump discontinuity of $\frac{d}{d k} \rho_{2}$ across $k=0$ to determine $\operatorname{cst}(\lambda)$ :

$$
\operatorname{cst}(\lambda)=\frac{\partial}{\partial k}\left[\sqrt{k} \mathrm{~J}_{\frac{1}{3}}\left(\frac{2}{3} \sqrt{\frac{\pi}{2 \lambda}}|k|^{\frac{3}{2}}\right)\right]_{k=-0}^{k=+0}=\left(\frac{\pi}{18 \lambda}\right)^{\frac{1}{6}} .
$$

Transforming back to $\rho(x, \lambda)$ and with the help of Eqs. (39) and (27) we get

$$
\rho(x, \lambda)=\sqrt{x} \int_{0}^{\infty} d k \cos (k \sqrt{x}) \sqrt{k} \mathrm{~J}_{\frac{1}{3}}\left(\frac{2}{3} \sqrt{\frac{\pi}{2 \lambda}}|k|^{\frac{3}{2}}\right)+\frac{\left(\frac{\pi}{18 \lambda}\right)^{\frac{1}{6}}}{\sqrt{x}} .
$$

Therefore the "time" dependent density reads,

$$
\tilde{\rho}(x, t)=\int_{0}^{\infty} d \lambda C(\lambda) \mathrm{e}^{-\lambda t} \rho(x, \lambda),
$$


where $C(\lambda)$ is determined by an initial condition.

Eqs. (42) and (43) will now be used to find the dynamical density-density correlation function. It is clear that $\operatorname{Corr}(x, y, t)$ and

$$
\operatorname{ICorr}(x, y, t):=\int_{0}^{y} d z \operatorname{Corr}(x, z, t)
$$

satisfy the same diffusion equation as $\rho(x, t)$. Hence,

$$
\operatorname{ICorr}(x, y, t)=\int_{0}^{\infty} d \lambda C(\lambda, y) \mathrm{e}^{-\lambda t} \rho(x, \lambda)
$$

and

$$
\begin{aligned}
\operatorname{Int}(x, y, t) & :=\int_{0}^{x} d z \operatorname{ICorr}(z, y, t) \\
& =\pi \int_{0}^{\infty} d \lambda C(\lambda, y) \mathrm{e}^{-\lambda t} \int_{0}^{\infty} d k \sqrt{k} \sin (k \sqrt{x}) J_{1 / 3}\left(\frac{2}{3}\left(\frac{\pi}{2 \lambda}\right)^{1 / 2} k^{3 / 2}\right),
\end{aligned}
$$

where we have used formula (74) in appendix A to arrive at the last equation. $C(\lambda, y)$ is determined by the "initial condition", $\operatorname{Int}(x, y, t=0)=\frac{1}{\beta \pi^{2}} \ln \left[\frac{|\sqrt{x}-\sqrt{y}|}{\sqrt{x}+\sqrt{y}}\right]$. A derivation of this formula can be found in appendix $\mathrm{B}$. To find $C(\lambda, y)$ we first perform a Fourier sine transform in the variable $\sqrt{x} ; \int_{0}^{\infty} d \sqrt{x} \sin (p \sqrt{x}) \cdots$, on Eq. (47) at $t=0$ to get an integral equation satisfied by $C(\lambda, y)$ 向

$$
-\frac{1}{\beta \pi p} \sin (p \sqrt{y})=\frac{\pi^{2}}{2} \int_{0}^{\infty} d \lambda C(\lambda, y) \sqrt{p} J_{1 / 3}\left(\frac{2}{3} \sqrt{\frac{\pi}{2 \lambda}} p^{3 / 2}\right)
$$

This can be rewritten as

$$
-\frac{2 \sin (p \sqrt{y})}{\beta \pi^{4} p^{3 / 2}}=\mathcal{H}_{1 / 3}\left(\frac{C\left(\frac{x}{2 u^{2}}, y\right)}{u^{4}}, \frac{2}{3} p^{3 / 2}\right)
$$

${ }^{6}$ We use the transform $\int_{0}^{\infty} d x \ln \left|\frac{x+a}{x-a}\right| \sin (p x)=\frac{\pi}{p} \sin (p a)$. 
where $\mathcal{H}_{\nu}(f(u), \xi)=\bar{f}_{\nu}(\xi):=\int_{0}^{\infty} d u f(u) u J_{\nu}(u \xi)$, denotes the Hankel transform. Using the Hankel inversion theorem $\left[f(x)=\int_{0}^{\infty} d \xi \bar{f}_{\nu}(\xi) \xi J_{\nu}(\xi u)\right.$, see[16], we find,

$$
\frac{C\left(\frac{\pi}{2 u^{2}}, y\right)}{u^{4}}=-\frac{4}{3 \beta \pi^{4}} \int_{0}^{\infty} d p \sin (p \sqrt{y}) \sqrt{p} J_{1 / 3}\left(\frac{2}{3} p^{3 / 2} u\right) .
$$

Therefore the "time" dependence of the twice integrated correlation function is displayed as a Laplace transform with the spectral parameter $\lambda:=\frac{\pi}{2 u^{2}}$,

$$
\operatorname{Int}(x, y, t)=-\frac{1}{3 \beta \pi^{2}} \int_{0}^{\infty} \frac{d \lambda}{\lambda^{2}} \mathrm{e}^{-\lambda t} F(x, \lambda) F(y, \lambda)
$$

where $F(x, \lambda):=\int_{0}^{x} d z \tilde{\rho}(z, \lambda)$ and $\tilde{\rho}(z, \lambda)$ given by Eqs. (27) and (42). Thus the variance of an arbitrary linear statistics,

$$
\mathcal{Q}(t):=\sum_{a} \mathcal{Q}\left(x_{a}(t)\right)=\int_{0}^{\infty} d x \sum_{a} \delta\left(x-x_{a}(t)\right) \mathcal{Q}(x)
$$

is

$$
\begin{gathered}
\operatorname{Var}[\mathcal{Q}, t]:=<\mathcal{Q}(t) \mathcal{Q}(0)>\mathrm{eq}-<\mathcal{Q}(t)>\mathrm{eq}<\mathcal{Q}(0)>\mathrm{eq} \\
=\int_{0}^{\infty} d x \int_{0}^{\infty} d y \mathcal{Q}(x) \mathcal{Q}(y) \operatorname{Corr}(x, y, t)=\int_{0}^{\infty} d x \int_{0}^{\infty} d y \mathcal{Q}^{\prime}(x) \mathcal{Q}^{\prime}(y) \operatorname{Int}(x, y, t) .
\end{gathered}
$$

This is the main result of our paper.

\section{Variance of $\frac{1}{1+x}$.}

As an example we take $\mathcal{Q}(x)=\frac{1}{1+x}$, which is the Landauer formula for the conductance. A simple calculation gives

$$
\operatorname{Var}\left[\frac{1}{1+x}, t\right]=\frac{\pi}{3 \beta} \int_{0}^{\infty} \frac{d \lambda}{\lambda^{2}} \mathrm{e}^{-\lambda t} h^{2}(\lambda)
$$

with

$$
h(\lambda):=\frac{1}{\pi^{2}} \int_{0}^{\infty} \frac{d x}{(1+x)^{2}} F(x, \lambda)
$$




$$
=\frac{1}{2} \int_{0}^{\infty} d k k^{3 / 2} \mathrm{e}^{-k} J_{1 / 3}\left(\frac{2}{3}\left(\frac{\pi}{2 \lambda}\right)^{1 / 2} k^{3 / 2}\right)=\left(\frac{2 \lambda}{\pi}\right)^{5 / 6} \sum_{n=0}^{\infty} A_{n}\left(\frac{18 \lambda}{\pi}\right)^{n / 3}
$$

where

$$
A_{n}:=\frac{(-1)^{n}}{n !} \frac{\Gamma(1+n / 3)}{\Gamma(-(n-1) / 3)}
$$

Eqs. (56) and (57) can be found in 17]. Now when $n \equiv 1 \bmod 3, A_{n}$ is taken to be zero.

The remaining terms can be grouped into two sums with $n \equiv 0 \bmod 3$ and $n \equiv 2 \bmod 3$ respectively. An application of the ratio test to these series shows that both converge absolutely and uniformly for all $\lambda$. We deduce that (with $\beta=2$ ),

$$
\frac{\pi}{6} \frac{h^{2}(\lambda)}{\lambda}=\lambda^{2 / 3} \sum_{n=0}^{\infty} B_{n} \lambda^{\frac{n}{3}}
$$

converges uniformly in the $\lambda^{1 / 3}$ plane. Using this representation and after performing the Laplace transform, we get,

$$
\operatorname{Var}\left[\frac{1}{1+x}, t\right]=\frac{1}{t^{2 / 3}} \sum_{n=0}^{\infty} B_{n} \frac{\Gamma\left(\frac{n+2}{3}\right)}{t^{\frac{n}{3}}}=\frac{C_{2 / 3}}{t^{2 / 3}}+\frac{C_{4 / 3}}{t^{4 / 3}}+\frac{C_{5 / 3}}{t^{5 / 3}}+\frac{C_{2}}{t^{2}}+\frac{C_{7 / 3}}{t^{7 / 3}}+\cdots,
$$

where

$$
\begin{aligned}
& C_{2 / 3}=\frac{\left(\frac{3}{2 \pi^{2}}\right)^{1 / 3} \Gamma(2 / 3)}{2 \Gamma^{2}(1 / 3)} \approx 0.050346 \\
& C_{4 / 3}=\frac{3\left(\frac{3}{2 \pi^{2}}\right)^{2 / 3} \Gamma(5 / 3) \Gamma(4 / 3)}{\Gamma(-1 / 3) \Gamma(1 / 3)} \approx-0.0632871 \text {, } \\
& C_{5 / 3}=-\frac{3\left(\frac{3}{2 \pi^{5}}\right)^{1 / 3} \Gamma(5 / 3)}{\Gamma(1 / 3) \Gamma(-2 / 3)} \approx 0.042735, \\
& C_{2}=\frac{27 \Gamma^{2}(5 / 3)}{4 \pi^{2} \Gamma^{2}(-1 / 3)} \approx 0.0337737, \\
& C_{7 / 3}=-\Gamma(7 / 3)\left[\frac{9\left(\frac{9}{4 \pi^{7}}\right)^{1 / 3} \Gamma(5 / 3)}{\Gamma(-1 / 3) \Gamma(-2 / 3)}-\frac{9\left(\frac{9}{4 \pi^{7}}\right)^{1 / 3} \Gamma(8 / 3)}{10 \Gamma(-4 / 3) \Gamma(1 / 3)}\right] \approx-0.0716263 .
\end{aligned}
$$


This completes the determination of the long "time" behaviour of $\operatorname{Var}[1 /(1+x), t]$. According to the Watson Lemma[18], Eq. (59) is an asymptotic expansion. The fact the Brownian motion ensemble tends to the stationary ensemble with exponential speed as soon as $\tau D / \gamma L \gg 1$, enables us to conclude that $\lim _{t \rightarrow \infty} \operatorname{Var}[\mathcal{Q}, t]=0$. At $t=0$, we simply go back to Eq.(55) and make use of the completeness theorem of the Hankel transform to show that $\operatorname{Var}[1 /(1+x), 0]=\frac{1}{8 \beta}$, a result obtained previously [19, 20, 3]. Other examples of $\mathcal{Q}$ can be found in 19 . 


\section{A Check of solution}

In this appendix we are going to show that

$$
\rho(x, \lambda)=\frac{\left(\frac{\pi}{18 \lambda}\right)^{\frac{1}{6}}}{\sqrt{x}}+\int_{0}^{\infty} \sqrt{x} \cos (k \sqrt{x}) \sqrt{k} \mathrm{~J}_{\frac{1}{3}}\left(\frac{2}{3} \sqrt{\frac{\pi}{2 \lambda}}|k|^{\frac{3}{2}}\right) d k
$$

is a solution to the integro-differential equation

$$
\lambda \rho(x, \lambda)=\frac{\partial}{\partial x} \frac{1}{\sqrt{x}} \int_{0}^{\infty} \frac{\rho(y, \lambda)}{x-y} d y
$$

To obtain better convergence in the manipulations we will instead show $\rho(x, \lambda)$ fulfills the equation obtained by integrating Eq.(61) from 0 to z. From the boundary condition

Eq.(26) we see that the lower boundary term on the right hand side to vanish. Using this,

Eq.(61) becomes

$$
\lambda \int_{0}^{z} d x \rho(x, \lambda)=\frac{1}{\sqrt{z}} \int_{0}^{\infty} \frac{\rho(y, \lambda)}{z-y} d y
$$

This equation is a stronger statement than Eq. (61) since any constant will vanish when Eq. (62) is differentiated to give Eq. (61). We start by evaluating the right hand side of Eq.(62),

$$
\begin{aligned}
\text { R.H.S } & =\frac{1}{\sqrt{z}} \int_{0}^{\infty} \frac{\rho(y, \lambda)}{z-y} d y \\
& =\frac{1}{\sqrt{z}} \int_{0}^{\infty} \frac{\left(\frac{\pi}{18 \lambda}\right)^{\frac{1}{6}}}{z-y} \frac{d y}{\sqrt{y}}+\frac{1}{\sqrt{z}} \int_{0}^{\infty} d y \int_{0}^{\infty} \frac{\sqrt{y} \cos (k \sqrt{y})}{z-y} \sqrt{k} \mathrm{~J}_{\frac{1}{3}}\left(\frac{2}{3} \sqrt{\frac{\pi}{2 \lambda}}|k|^{\frac{3}{2}}\right) d k \\
& =\frac{1}{\sqrt{z}} \int_{0}^{\infty} d y \int_{0}^{\infty} \frac{\sqrt{y} \cos (k \sqrt{y})}{z-y} \sqrt{k} \mathrm{~J}_{\frac{1}{3}}\left(\frac{2}{3} \sqrt{\frac{\pi}{2 \lambda}}|k|^{\frac{3}{2}}\right) d k
\end{aligned}
$$

Using the identity,

$$
\int_{0}^{\infty} \frac{\sqrt{y} \cos (k y)}{z-y} d y=2 \pi \delta(k)+\pi \sqrt{z} \sin (k \sqrt{z})
$$


in Eq. (64) gives

$$
\frac{1}{\sqrt{z}} \int_{0}^{\infty} \frac{\rho(y, \lambda)}{z-y} d y=\pi \int_{0}^{\infty} \sin (k \sqrt{z}) \sqrt{k} \mathrm{~J}_{\frac{1}{3}}\left(\frac{2}{3} \sqrt{\frac{\pi}{2 \lambda}}|k|^{\frac{3}{2}}\right) d k .
$$

We now turn to the left hand side of Eq. (62)

$$
\begin{aligned}
\text { L.H.S } & =\lambda \int_{0}^{z} d x \rho(x, \lambda) \\
& =\lambda \int_{0}^{z} \frac{\left(\frac{\pi}{18 \lambda}\right)^{\frac{1}{6}} d x}{\sqrt{x}}+\lambda \int_{0}^{z} d x \int_{0}^{\infty} \sqrt{x} \cos (k \sqrt{x}) \sqrt{k} \mathrm{~J}_{\frac{1}{3}}\left(\frac{2}{3} \sqrt{\frac{\pi}{2 \lambda}}|k|^{\frac{3}{2}}\right) d k \\
& =2 \lambda\left(\frac{\pi}{18 \lambda}\right)^{\frac{1}{6}} \sqrt{z}+2 \lambda \int_{0}^{\infty} \int_{0}^{\sqrt{z}} d t t^{2} \cos (k t) \sqrt{k} \mathrm{~J}_{\frac{1}{3}}\left(\frac{2}{3} \sqrt{\frac{\pi}{2 \lambda}}|k|^{\frac{3}{2}}\right) d k .(67)
\end{aligned}
$$

The integral with respect to $t=\sqrt{x}$ is

$$
\int_{0}^{\sqrt{z}} d t t^{2} \cos (k t)=-\frac{\partial^{2}}{\partial k^{2}}\left\{\frac{\sin (k \sqrt{z})}{k}\right\} .
$$

The left hand side is then

$$
\begin{aligned}
\text { L.H.S }= & 2 \lambda\left(\frac{\pi}{18 \lambda}\right)^{\frac{1}{6}} \sqrt{z}-2 \lambda \int_{0}^{\infty} \frac{\partial^{2}}{\partial k^{2}}\left\{\frac{\sin (k \sqrt{z})}{k}\right\} \sqrt{k} \mathrm{~J}_{\frac{1}{3}}\left(\frac{2}{3} \sqrt{\frac{\pi}{2 \lambda}}|k|^{\frac{3}{2}}\right) d k \\
= & 2 \lambda\left(\frac{\pi}{18 \lambda}\right)^{\frac{1}{6}} \sqrt{z}-2 \lambda\left[\frac{\partial}{\partial k}\left\{\frac{\sin (k \sqrt{z})}{k}\right\} \sqrt{k} \mathrm{~J}_{\frac{1}{3}}\left(\frac{2}{3} \sqrt{\frac{\pi}{2 \lambda}}|k|^{\frac{3}{2}}\right)\right]_{0}^{\infty} \\
& +2 \lambda \int_{0}^{\infty} \frac{\partial}{\partial k}\left\{\frac{\sin (k \sqrt{z})}{k}\right\} \frac{\partial}{\partial k}\left\{\sqrt{k} \mathrm{~J}_{\frac{1}{3}}\left(\frac{2}{3} \sqrt{\frac{\pi}{2 \lambda}}|k|^{\frac{3}{2}}\right)\right\} d k .
\end{aligned}
$$

The boundary terms from the partial integration are zero, since

$$
\begin{gathered}
\sqrt{k} \mathrm{~J}_{\frac{1}{3}}\left(\frac{2}{3} \sqrt{\frac{\pi}{2 \lambda}}|k|^{\frac{3}{2}}\right) \sim \operatorname{const} k^{-\frac{1}{4}} \cos \left(\text { const } k^{\frac{3}{2}}-\text { phase }\right), k \rightarrow \infty \\
=0, \quad k=0 .
\end{gathered}
$$

Now,

$$
\begin{aligned}
\text { L.H.S }= & 2 \lambda\left(\frac{\pi}{18 \lambda}\right)^{\frac{1}{6}} \sqrt{z}+2 \lambda\left[\frac{\sin (k \sqrt{z})}{k} \frac{\partial}{\partial k}\left\{\sqrt{k} \mathrm{~J}_{\frac{1}{3}}\left(\frac{2}{3} \sqrt{\frac{\pi}{2 \lambda}}|k|^{\frac{3}{2}}\right)\right\}\right]_{0}^{\infty} \\
& -2 \lambda \int_{0}^{\infty} \frac{\sin (k \sqrt{z})}{k} \frac{\partial^{2}}{\partial k^{2}}\left\{\sqrt{k} \mathrm{~J}_{\frac{1}{3}}\left(\frac{2}{3} \sqrt{\frac{\pi}{2 \lambda}}|k|^{\frac{3}{2}}\right)\right\} d k .
\end{aligned}
$$


The boundary term at infinity vanishes. The boundary term at the origin is

$$
-\left.2 \lambda \sqrt{z} \frac{\partial}{\partial k}\left\{\sqrt{k} \mathrm{~J}_{\frac{1}{3}}\left(\frac{2}{3} \sqrt{\frac{\pi}{2 \lambda}}|k|^{\frac{3}{2}}\right)\right\}\right|_{k=0}=-2 \lambda\left(\frac{\pi}{18 \lambda}\right)^{\frac{1}{6}} \sqrt{z}
$$

By differentiation,

$$
\frac{1}{k} \frac{\partial^{2}}{\partial k^{2}}\left\{\sqrt{k} \mathrm{~J}_{\frac{1}{3}}\left(\frac{2}{3} \sqrt{\frac{\pi}{2 \lambda}}|k|^{\frac{3}{2}}\right)\right\}=-\frac{\pi}{2 \lambda} \sqrt{k} \mathrm{~J}_{\frac{1}{3}}\left(\frac{2}{3} \sqrt{\frac{\pi}{2 \lambda}}|k|^{\frac{3}{2}}\right) .
$$

Gathering the pieces we have

$$
\begin{aligned}
\lambda \int_{0}^{z} d x \rho(x, \lambda) & =\pi \int_{0}^{\infty} \sin (k \sqrt{z}) \sqrt{k} \mathrm{~J}_{\frac{1}{3}}\left(\frac{2}{3} \sqrt{\frac{\pi}{2 \lambda}}|k|^{\frac{3}{2}}\right) d k \\
\lambda \int_{0}^{z} d x \rho(x, \lambda) & =\frac{1}{\sqrt{z}} \int_{0}^{\infty} \frac{\rho(y, \lambda)}{z-y} d y
\end{aligned}
$$

Here we make two remarks on the above derivations:

1. Since the number of particles in the interval $(0, z)$ goes to zero, when $z$ tends to zero, the last equation shows that the zero flux condition at the origin is fulfilled.

2. This is concern with the $z \rightarrow \infty$ limit in Eq. (74). The integrand on the right hand side is the product of two strongly oscillating functions, which oscillate out of phase. Since none of them are absolutely integrable, a Riemann-Lebesque lemma can not be used to conclude that the integral tends to zero. We may appeal to the theory of generalized functions that it is 0. The R. H. S. of Eq. (74) can be re-written up to irrelevant constants as

$$
\mathcal{J}(a)=\int_{0}^{\infty} d y \sin (y a) \sqrt{y} J_{1 / 3}\left(b y^{3 / 2}\right)
$$

where $a:=\sqrt{z}$ and $b:=\frac{2}{3}\left(\frac{\pi}{2 \lambda}\right)^{1 / 2}$. We wish to determine $\mathcal{J}(a)$ in the limit $a \rightarrow \infty$, with $b(>0)$ fixed. Consider instead,

$$
\mathcal{J}_{\mu}(a)=\int_{0}^{\infty} d y \frac{\sin (y a)}{y} y^{1-\mu} J_{1 / 3}\left(b y^{3 / 2}\right), \quad-\frac{5}{4}<\Re \mu<\frac{5}{2} .
$$


The integral we need can be defined as the analytic continuation of $\mathcal{J}_{\mu}(a)$ to $\mu=-1 / 2$. Now, since $\lim _{a \rightarrow \infty} \frac{\sin (a x)}{x}=\delta(x)$, we conclude by integrating over the $\delta$ function that $\lim _{a \rightarrow \infty} \mathcal{J}_{\mu}(a)=0$. Hence $\mathcal{J}(\infty)=0$. Physically this means that the total number of particles in each lambda mode is zero. The zero mode, of course, contains particles. 


\section{B The equal time variance of a linear statistic.}

The equal time variance of a linear statistic $\mathcal{Q}$ is

$$
\operatorname{Var}(\mathcal{Q}, 0)=\int_{0}^{\infty} d x \int_{0}^{\infty} d y \mathcal{Q}(x) \mathcal{Q}(y) \operatorname{Corr}(x, y, 0)
$$

a result first derived in [19] by the method of functional derivative. The claim in [19] is that the method is valid for all potentials, however, this is flawed by the use of the $N=\infty$ density, which may not exist for all potentials. For the sake of completeness, we present in this appendix a small modification of the arguments given in [19] and show that the result has a more general validity. Instead of focusing on Eq. (76), we prefer to work with the partially integrated expressions

$$
\operatorname{Var}(\mathcal{Q}, t)=-\int_{0}^{\infty} d x \int_{0}^{\infty} d y \mathcal{Q}(x) \mathcal{Q}^{\prime}(y) \operatorname{ICorr}(x, y, t)
$$

and

$$
\operatorname{Var}(\mathcal{Q}, t)=\int_{0}^{\infty} d x \int_{0}^{\infty} d y \mathcal{Q}^{\prime}(x) \mathcal{Q}^{\prime}(y) \operatorname{Int}(x, y, t), \quad 7
$$

where

$$
\begin{aligned}
\operatorname{ICorr}(x, y, t) & \equiv \int_{0}^{y} d z \operatorname{Corr}(x, z, t) \\
\operatorname{Int}(x, y, t) & \equiv \int_{0}^{x} d z \operatorname{ICorr}(z, y, t)=\int_{0}^{x} d z_{1} \int_{0}^{y} d z_{2} \operatorname{Corr}\left(z_{1}, z_{2}, t\right)
\end{aligned}
$$

We will start by finding $\operatorname{ICorr}(x, y, 0)$. By definition $\sigma_{\text {gas }}(x)=\sum_{n=1}^{\infty} \delta\left(x-x_{n}^{0}\right)$ and

$$
<\sigma_{\operatorname{gas}}(x)>_{\text {eq }}=\frac{\int_{0}^{\infty} d x_{1}^{0} \ldots \int_{0}^{\infty} d x_{N}^{0} \sigma_{\operatorname{gas}}(x) e^{-W[u]}}{\int_{0}^{\infty} d x_{1}^{0} \ldots \int_{0}^{\infty} d x_{N}^{0} e^{-W[u]}} .
$$

\footnotetext{
${ }^{7}$ To avoid the boundary terms it has been assumed that the linear statistic fulfills $\left.\sqrt{x} \mathcal{Q}(x)\right|_{x=0}=0$. The linear statistic corresponding to the conductance $\frac{1}{1+x}$ satisfies this criterion.
} 
The dependence of the energy, $W$, on the coordinates $x_{1}^{0}, x_{2}^{0}, \ldots, x_{N}^{0}$ has been suppressed. The external potential $u(x)$ is assumed to be bounded at the origin which is the physically interesting situation. Now write $u(x)=-\int_{0}^{x} f(z) d z+u(0)$, where $f(x)$ is the force. The constant $u(0)$ can be set to zero as this can always be accomplished by a redefinition of the zero point energy. The energy is now

$$
W[u]=W\left[-\int_{0}^{x} f(z) d z\right]=-\sum_{n=1}^{N} \int_{0}^{\infty} f(z) \theta\left(x_{n}^{0}-z\right) d z-\sum_{i<j} \ln \left|x_{j}^{0}-x_{i}^{0}\right|
$$

where $\theta$ is the Heaviside step function. The functional derivative of $W\left[-\int_{0}^{x} f(z) d z\right]$ with respect to $f$ is

$$
\frac{\delta W\left[-\int_{0}^{x} f(z) d z\right]}{\delta f(y)}=-\sum_{n=1}^{\infty} \theta\left(x_{n}^{0}-y\right)=-\int_{0}^{y} \sigma_{\operatorname{gas}}(x, 0) d z .
$$

The functional derivative of $\sigma_{\mathrm{eq}}(x)=<\sigma_{\text {gas }}(x, 0)>$ eq with respect to $f$ is recognized as $\operatorname{ICorr}(x, y, 0)$,

$$
\operatorname{ICorr}(x, y, 0)=\frac{\delta \sigma_{\mathrm{eq}}(x)}{\beta \delta f(y)}
$$

The density $\sigma_{\mathrm{eq}}(x)$ is approximated by

$$
\sigma(x, N ; f)=-\frac{1}{\pi^{2}} \sqrt{\frac{b(N, f)-x}{x}} \int_{0}^{b(N, f)} \frac{d z}{z-x} \sqrt{\frac{z}{b(N, f)-z}} f(z),
$$

where $b(N, f)$ is the upper limit of support of $\sigma(x, N ; f) . \frac{\delta \sigma(x, N ; f)}{\delta f(y)}$ is computed as the linear term in $\epsilon$, when $\epsilon \delta(x-y)$ is added to the force $f(x)$. We have

$$
\begin{aligned}
\sigma(x, N ; f+\epsilon \delta(x-y)) & =-\frac{1}{\pi^{2}} \sqrt{\frac{b-x}{x}} \int_{0}^{b} \frac{d z}{z-x} \sqrt{\frac{z}{b-z}}[f(z)+\epsilon \delta(z-y)] \\
& =\epsilon \frac{1}{\pi^{2}} \sqrt{\frac{y}{x}} \frac{1}{x-y} \sqrt{\frac{b-x}{b-y}}+\sigma(x, N-\eta ; f)
\end{aligned}
$$

where $\eta=\eta(\epsilon, y, N, f)=\epsilon \frac{1}{\pi^{2}} \int_{0}^{b} \sqrt{\frac{y}{x}} \frac{1}{x-y} \sqrt{\frac{b-x}{b-y}} d x=-\epsilon \frac{1}{\pi} \frac{y}{b-y}$ is the number of particles associated with the force $\epsilon \delta(x-y)$ and $b:=b(N, f+\epsilon \delta(x-y))=b(N-\eta, f)$ is the upper 
limit of support of $\sigma(x ; N, f+\epsilon \delta(x-y))$ and $\sigma(x, N-\eta ; f)$. The functional derivative is

$$
\frac{\delta \sigma(x, N)}{\beta \delta f(y)}=\frac{1}{\beta \pi^{2}} \sqrt{\frac{y}{x}} \frac{1}{x-y} \frac{b-x}{b-y}+\frac{1}{\beta \pi} \frac{y}{b-y} \frac{\partial \sigma(x, N)}{\partial N} .
$$

In the limit $N \rightarrow \infty, \sqrt{\frac{b-x}{b-y}}=1, \eta$ and $\frac{\partial \sigma(x, N)}{\partial N}$ tend to zero. The extra density, $\Delta \sigma(x)$, when $\epsilon$ particles is added to the system, experiences no force in the interval $(0, b)$. Therefore $\Delta \sigma(x)$ is less than the corresponding density with $\epsilon$ particles in the box $(0, b)$ : $\frac{1}{\pi} \frac{\epsilon}{\sqrt{x(b-x)}}, \quad x \in(0, b)$. Outside $(0, b), \Delta \sigma(x)$ tends monotonically to zero. We conclude, in this limit, the functional derivative is reduced to

$$
\begin{aligned}
\frac{\delta \sigma(x, N)}{\beta \delta f(y)} & =\frac{1}{\beta \pi^{2}} \sqrt{\frac{y}{x}} \frac{1}{x-y} \\
\operatorname{ICorr}(x, y, 0) & =\frac{1}{\beta \pi^{2}} \frac{\partial}{\partial x} \ln \left|\frac{\sqrt{x}-\sqrt{y}}{\sqrt{x}+\sqrt{y}}\right| .
\end{aligned}
$$

From this it is seen that

$$
\operatorname{Int}(x, y, 0)=\frac{1}{\beta \pi^{2}} \ln \left|\frac{\sqrt{x}-\sqrt{y}}{\sqrt{x}+\sqrt{y}}\right|
$$

and

$$
\operatorname{Var}(\mathcal{Q}, 0)=\frac{1}{\beta \pi^{2}} \int_{0}^{\infty} d x \int_{0}^{\infty} d y \mathcal{Q}^{\prime}(x) \mathcal{Q}^{\prime}(y) \ln \left|\frac{\sqrt{x}-\sqrt{y}}{\sqrt{x}+\sqrt{y}}\right|
$$

Thus at equal time, variances are independent of the potential $u$.

${ }^{8}$ They have the same upper limit, because the part of $\sigma(x, N ; f+\epsilon \delta(x-y))$ corresponding to $f$ : $-\frac{1}{\pi^{2}} \sqrt{\frac{b-x}{x}} \int_{0}^{b} \frac{d z}{z-x} \sqrt{\frac{z}{b-z}} f(z)$ is a solution, with $N-\eta$ particles, to the problem with the external force $f$, obeying the right boundary conditions. In other words $-\frac{1}{\pi^{2}} \sqrt{\frac{b-x}{x}} \int_{0}^{b} \frac{d z}{z-x} \sqrt{\frac{z}{b-z}} f(z)=\sigma(x, N-\eta ; f)$. 


\section{References}

[1] M. L. Mehta, 1991 Random Matrices 2nd edn (New York: Academic)

[2] A D Stone, P A Mello, K A Muttalib and J-L Pichard, 1991 Mesoscopic Phenomena in Solids ed B L Altshuler, P A Lee and R A Webb (Amsterdam:North-Holland)

[3] The $\alpha$ - ensemble was introduced in Y Chen and S M Manning, J. Phys.: Condens. Matter 6 (1994) 3039. And the level spacing distribution was investigated by[四].

[4] Y Chen and K J Eriksen, Gap Formation Probability of the $\alpha$ - ensemble, 1994 preprint submitted to J. Phys. A, cond-mat@babbage.sissa.it/9409017.

[5] The terminology of hard edge is that of C A Tracy and H Widom, Commun. Math. Phys. 161 (1994) 289. See also ibid., 163 (1994) 33.

[6] A Szafer and B L Altshuler, Phys. Rev. Lett. 70 (1993) 587.

[7] C W J Beenakker, Phys. Rev. Lett. 70 (1993) 4126.

[8] F J Dyson, J. Math. Phys. 13 (1972) 90.

[9] B D Simons and B L Altshuler, Phys. Rev. Lett. 70 (1993) 4063; B D Simons, A Szafer and B L Altshuler, Pis'ma Zh. Eksp. Teor. Fiz. 57 (1993) 268[JETP Lett. 57 (1993) 276]

[10] K B Efetov, Adv. Phys. 32 (1983) 53.

[11] C W J Beenakker and B Rejaei, Physica A 203 (1994) 61. 
[12] A M S Macedo, Universal parametric correlations in the transmission eigenvalue spectra disordered conductors, 1994, cond-mat @ babbage. sissa. it /9404055. This paper studies the parametric correlations of the transmission eigenvalues (supported on the right half-line) of disordered conductors. However, in this formulation the eigenvalue density has no hard edge singularity; $\sigma(x) \sim$ constant, $x \sim 0$.

[13] Y. Chen and S. M. Manning, J. Phys. A: Math. gen. 27 (1994) 3615.

[14] The asymptotic gap formation probability reads,

$$
E_{\beta}(0, s) \sim G(1+\alpha) \frac{\mathrm{e}^{-\frac{s}{2 \beta}+\frac{2 \alpha \sqrt{s}}{\beta}}}{s^{\frac{\alpha(2-\beta)}{4 \beta}+\frac{\alpha^{2}}{2 \beta}}}\left[1+\mathrm{O}\left(\frac{1}{\sqrt{s}}\right)\right]
$$

where $G($.$) is the Barnes G$-function.

[15] N I Akhiezer and I M Glazman, Theory of Linear Operator in Hilbert Space,vol 1 (New York:Ungar).

[16] I N Sneddon, 1979, The Use of Integral Transforms, (MacGraw-Hill)

[17] Formula 2.12.1.5 of A P Prudnikov, Yu A Brychkov and O I Marichev, Integral and Series, volume 2, 1986, (Gordon and Breach).

[18] See for example, J D Murray, Asymptotic Analysis, 1974, (Clarendon:Oxford)

[19] C W J Beenakker, Phys. Rev. Lett. 70 (1993) 1155.

[20] E L Basor and C A Tracy, J. Stat. Phys. 73 (1993) 415. 